Fortschritt-Berichte VDI

VDI

Reihe 8

Mess-,

Steuerungs- und

Regelungstechnik

Nr. 1245
Dipl.-Inform. Henning Mersch, Bielefeld

Deterministische, dynamische Systemstrukturen in der Automatisierungstechnik

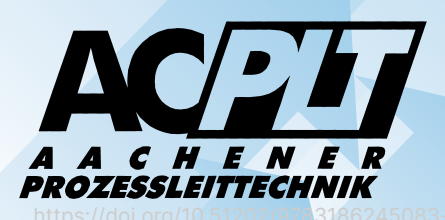

Lehrstuhl für

Prozessleittechnik

der RWTH Aachen 
https://doi.org/10.51202/9783186245083-1

Generiert durch IP '172.22.53.54', am 26.04.2023, 04:11:05

Das Erstellen und Weitergeben von Kopien dieses PDFs ist nicht zulässig. 


\title{
Deterministische, dynamische Systemstrukturen in der Automatisierungstechnik
}

\author{
Von der Fakultät für Georessourcen und Materialtechnik \\ der Rheinisch-Westfälischen Technischen Hochschule Aachen \\ zur Erlangung des akademischen Grades eines \\ Doktors der Ingenieurwissenschaften \\ genehmigte Dissertation \\ vorgelegt von Dipl.-Inform.
}

Henning Mersch

aus Bielefeld

Berichter: Univ.-Prof. Dr.-Ing. Ulrich Epple

Univ.-Prof. Dr.-Ing. habil. Martin Wollschlaeger

Tag der mündlichen Prüfung: 26. November 2015 
https://doi.org/10.51202/9783186245083-1

Generiert durch IP '172.22.53.54', am 26.04.2023, 04:11:05

Das Erstellen und Weitergeben von Kopien dieses PDFs ist nicht zulässig. 


\section{Fortschritt-Berichte VDI}

\section{Reihe 8}

Mess-, Steuerungsund Regelungstechnik

Nr. 1245
Dipl.-Inform. Henning Mersch, Bielefeld

Deterministische, dynamische Systemstrukturen in der Automatisierungstechnik

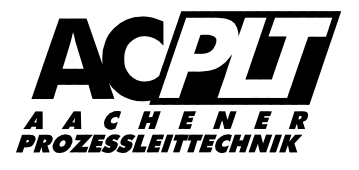

Lehrstuhl für

Prozessleittechnik

der RWTH Aachen 
Mersch, Henning

\section{Deterministische, dynamische Systemstrukturen in der Automatisierungs- technik}

Fortschr.-Ber. VDI Reihe 8 Nr. 1245. Düsseldorf: VDI Verlag 2016.

142 Seiten, 77 Bilder, 3 Tabellen.

ISBN 978-3-18-524508-4, ISSN 0178-9546,

€ 52,00/VDI-Mitgliederpreis € 46,80.

Für die Dokumentation: Anlagen - Automatisierungstechnik - Systemstruktur - Modelle Verteilte Systeme - Dynamik - Nachvollziehbarkeit - Flexibilität - OPC-UA

Für die Weiterentwicklung der Automatisierungstechnik ist die erweiterte Zusammenarbeit der automatisierungstechnischen Geräte wichtig. Viele aktuelle Themen, wie "Industrie 4.0" oder "Cyber Physical Systems" gehen davon aus, dass Informationen aus dem Engineering zur Produktionszeit bereit stehen. In der Automatisierungstechnik wird dafür immer mehr angestrebt, Modelle zur Beschreibung der unterschiedlichsten Sachverhalte zu nutzen. Dabei werden Themen-spezifische Modelle entwickelt, die unabhängig voneinander eigene Blickwinkel der Automatisierungstechnik auf eine Anlage beschreiben. Die vorliegende Arbeit beschreibt Mittel, um diese Modelle zur Anwendung zu bringen, verzichtet dabei aber auf ein zentralistisches Modell: Existierende, heterogene Modelle werden durch eine verteilte, dynamische Ausführungsumgebung für Modelle und Dienste in der Automatisierungstechnik nutzbar gemacht, welche kollaborative Ansätze zur gemeinsamen Datenhaltung auf unterschiedlichen Geräten ermöglichen.

\section{Bibliographische Information der Deutschen Bibliothek}

Die Deutsche Bibliothek verzeichnet diese Publikation in der Deutschen Nationalbibliographie; detaillierte bibliographische Daten sind im Internet unter http://dnb.ddb.de abrufbar.

\section{Bibliographic information published by the Deutsche Bibliothek}

(German National Library)

The Deutsche Bibliothek lists this publication in the Deutsche Nationalbibliographie (German National Bibliography); detailed bibliographic data is available via Internet at http://dnb.ddb.de.

Alle Rechte, auch das des auszugsweisen Nachdruckes, der auszugsweisen oder vollständigen Wiedergabe (Fotokopie, Mikrokopie), der Speicherung in Datenverarbeitungsanlagen, im Internet und das der Übersetzung, vorbehalten. 


\section{Vorwort}

Diese Dissertation entstand durch meine Tätigkeit als wissenschaftlicher Angestellter am Lehrstuhl für Prozessleittechnik der RWTH Aachen. An dieser Stelle möchte ich mich bei denen bedanken, die zum Gelingen der Arbeit beigetragen haben.

Mein besonderer Dank gilt Herrn Professor Dr.-Ing. Ulrich Epple. Die von ihm am Lehrstuhl geschaffene, offene und angenehme Arbeitsatmosphäre in einer konstruktiven und gut ausgestatteten Umgebung sind der Ausgangspunkt für diese Arbeit. Die fachlichen und teilweise auch kontroversen Diskussionen waren immer erfrischend und inspirierend für weitere Arbeiten.

Ebenso bedanke ich mich bei Herrn Professor Dr.-Ing. habil. Martin Wollschlaeger, Inhaber der Professur Prozesskommunikation der Technischen Universität Dresden, für die freundliche Übernahme der Rolle des Zweitgutachters.

Für intensive Diskussionen und die kooperative Arbeitsatmosphäre danke ich weiterhin meinen ehemaligen Kollegen, den Mitarbeiterinnen und Mitarbeitern des Lehrstuhls. Besonders erwähnen möchte ich hier Reiner Jorewitz, Martin Mertens, Gustavo Quirós und Markus Schlüter

Für die vielen organisatorischen Arbeiten gilt Martina Uecker und im Sekretariat Frau Bey der besondere Dank. Nicht unerwähnt bleiben sollen auch die vielen studentischen Hilfskräfte, die viele Tätigkeiten erst umsetzbar machen.

Weiterhin möchte ich gerne Leon Urbas, Andreas Gössling, Christian Kleegrewe und Wolfgang Mahnke danken, die immer wieder erfrischende Gedanken in privaten Gesprächen oder der gemeinsamen VDI-GMA Fachausschussarbeit geweckt haben.

Meiner Frau Tina danke ich von ganzem Herzen für die Geduld und die Zeit sowie den fachlichen Diskussionen und lektographischen Anmerkungen. Unserem Sohn Liam muss ich für so manche Stunde danken, die er schon in den frühen Jahren in Geduld üben musste.

Schließlich danke ich meinen Eltern Birgit und Werner Mersch, die mich zu diesem beruflichen und privaten Weg geführt haben und in vielen Situationen zum Gelingen dieser Arbeit beigetragen haben.

Verl, im Dezember 2015

Henning Mersch 
"So you do what you do best. And you link to the rest."

Jeff Jarvis, 22. Februar 2007 [Jar] 


\section{Inhaltsverzeichnis}

1 Einleitung 1

1.1 Ziele und Vision . . . . . . . . . . . . . . . . . . . 3

1.2 Konzepte der verteilten, modellgetriebenen Instanzumgebung . . . . . . . . . . . . . . 4

1.3 Übersicht des Vorgehens . . . . . . . . . . . . . . . . . . . . . . . 8

2 Stand der Wissenschaft und Technik - mit Begriffsklärung 10

2.1 Vom Wissen zu Maschinen-verarbeitbaren Modellen in der AT . . . . . . . . . . . 10

2.1 .1 (Modell-)Relationen . . . . . . . . . . . . . . . . . . . . . . 12

2.1.2 Instanz-Struktur: Komponenten als Gruppierung . . . . . . . . . . . . . 14

2.1.3 Bestandteile einer Modell-Beschreibung . . . . . . . . . . . . . . . . 15

2.2 Existierende Modelle der Automatisierungstechnik . . . . . . . . . . . . . . . . . . . . . . . . . . .

2.3 Kommunikation in der Automatisierungstechnik . . . . . . . . . . . . . . . . 18

2.3.1 Kommunikations-Medien: Bussysteme und Alternativen . . . . . . . . . . . 19

2.3.2 Formen der Kommunikation . . . . . . . . . . . . . . . . . . . . . 21

2.3.3 Kommunikations-Systeme für den Zugriff auf Modelle . . . . . . . . . . . 22

2.4 Instanzumgebung der Modelle . . . . . . . . . . . . . . . . . . . . 24

2.4.1 Existierende Instanzumgebungen für Modelle in die AT . . . . . . . . . 25

2.4.1.1 ACPLT-Technologien . . . . . . . . . . . . . . . . . . 26

2.4 .1 .2 OPC-UA . . . . . . . . . . . . . . . . . 26

2.4.2 Aktive Komponenten im Modell: Dienste . . . . . . . . . . . . . . . . . 27

2.4.2.1 Dienste - ein Versuch der Erfassung des Begriffes . . . . . . . . 28

2.4.3 Existierende Ausführungsumgebungen für Dienste . . . . . . . . . . . . . 31

2.4.4 Aspekte von Anwendungen, Diensten und Apps . . . . . . . . . . . . . . 32

2.4.5 Existierende Ausführungsumgebungen für Modelle und Dienste . . . . . 35

2.5 Verteilte Systeme . . . . . . . . . . . . . . . . . . . 35

3 Analyse der Anforderungen $\quad 37$

3.1 Ergänzende Anforderungen an Geräte und Umgebung . . . . . . . . . . . . . . 37

3.2 Ergänzende Anforderungen an Meta-Modell und Instanzumgebung . . . . . . . . 37

3.3 Ergänzende Anforderungen an die Kommunikation . . . . . . . . . . . . . . . . . . 39

3.3.1 Einheitliche, allgemeine Adressierung . . . . . . . . . . . . . . . 40

3.4 Bezug der Anforderungen . . . . . . . . . . . . . . . . . . . . 41

3.5 Nachvollziehbarkeit und Verständlichkeit . . . . . . . . . . . . . . . . . . . 42 
4 Modell-Architektur für dynamische, verteilte Systemstrukturen $\quad \mathbf{4 3}$

4.1 Beispiel-Modell: AT-Geräte-Struktur . . . . . . . . . . . . . . . . . . . 43

4.1 .1 Erweiterung: Routing . . . . . . . . . . . . . . . . . . . . . 44

4.2 Abbildung der Realität: Repräsentationen im Modell . . . . . . . . . . . . . . . . . . . . . . . . . . 46

4.2.1 Zustandsmaschine für aktive Komponenten . . . . . . . . . . . . . . . . . 46

4.2 .2 Die Komponenten-Repräsentation . . . . . . . . . . . . . . . . . . . . . 48

4.2.3 Unspezifizierte, flexible Annotationen für Repräsentationen . . . . . . . . . 50

4.2.4 Unterschiedliche Komponenten-Repräsentationen . . . . . . . . . . . . . 52

4.2.5 Beispiel: AT-Geräte-Struktur als Komponenten-Repräsentation . . . . . . 53

4.3 Kommunikation im automatisierungstechnischen Kontext . . . . . . . . . . . . . 54

4.3.1 Referenzierung über Systemgrenzen hinweg . . . . . . . . . . . . . 55

4.3.2 Kommunikations-Medien . . . . . . . . . . . . . . . . . 57

4.3.3 Nachrichten-basierte Kommunikation . . . . . . . . . . . . . . . 58

4.3.4 Typ 1: singuläre Kommunikation . . . . . . . . . . . . . . . . 62

4.3.5 Typ 2: Aufruf/Antwort Kommunikation . . . . . . . . . . . . . . . 62

4.3.6 Typ 3: Subskription/Benachrichtigungs-Kommunikation . . . . . . . . . 62

4.3.7 Typ 4: Indirekte Kommunikation per Intents . . . . . . . . . . . . . . . . . 64

4.3.8 Lokale Kommunikation . . . . . . . . . . . . . . . . . . . . . . . 66

4.4 Dienst-Modell: aktive, dynamische Komponenten . . . . . . . . . . . . . . . . 67

4.5 Die modellgetriebene Instanzumgebung . . . . . . . . . . . . . . . . . . . . . 69

4.5.1 Vom Modell zum Instanz-Modell . . . . . . . . . . . . . . . . . 70

4.5.1.1 Modell-Master . . . . . . . . . . . . . . . 71

4.5 .2 Sprache der Modell-Änderungen . . . . . . . . . . . . . . . . . 72

4.5 .3 Änderungs-Benachrichtigungen . . . . . . . . . . . . . . . . . . . . . . . . . . . . 73

4.5.3.1 Alternative Realisierung: Intents . . . . . . . . . . . . . 75

4.5.4 Ausführungsumgebung: Dienste als partielle, Aufgaben-orientierte Teil-

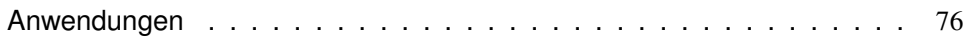

4.6 Modell- und Gerätegrenzen . . . . . . . . . . . . . . . . . . . . . . . 77

4.6.1 Modelle in Relation: Modell-Interkonnektion . . . . . . . . . . . . . . . . 77

4.6.1.1 Beispiel: AT-Geräte-Struktur und Anlagenstruktur . . . . . . . . . 79

4.6.2 Über Gerätegrenzen hinweg: Verteilte (Modell-)Laufzeiten . . . . . . . . . . 80

4.6.2.1 Externe Verbindungen . . . . . . . . . . . . . . . . . . . . . . . . 80

4.6.2.2 Änderungs-Benachrichtigungen . . . . . . . . . . . . . . . . . 83

4.6.2.3 Zugriff auf die verteilte Modell-Instanzen . . . . . . . . . . . . . 83

4.6.2.4 Dienst-Orchestrierung auf Basis der verteilten Modelle . . . . . 86

4.6.2.5 Beispiel: Verteilte Modellierung der AT-Geräte-Struktur . . . . . 87

4.6.2.6 Transparenter Zugriff auf verteilte Modell-Instanzen . . . . . . . 87

4.7 Effizienz der Konzepte . . . . . . . . . . . . . . . . . . . . . . . . . . . . 89

4.7.1 Modell-Interkonnektionen und ihre Etablierung . . . . . . . . . . . . . . 89

4.7 .2 Verteilungsaspekte . . . . . . . . . . . . . . . . . . . . 90

4.8 Integrationsmöglichkeiten in die bestehende AT-Geräte-Landschaft . . . . . . . . 91

5 Komponenten einer verteilten, modellgetriebenen Ausführungsumgebung 92

5.1 Ressourcen-Abstraktion: IMLAUF-Kern . . . . . . . . . . . . . . . . . . . . 95 
5.2 Die modellgetriebene Instanzumgebung . . . . . . . . . . . . . . . . . . . . 97

5.3 Nachrichten-basierte Kommunikation in IMLAUF: MsgSys . . . . . . . . . . . . . 98

5.4 Transparente Erkundung von Daten - Modell-Explorer . . . . . . . . . . . . . . 100

5.5 Prinzip der Modell-Interkonnektions-Komponenten (MIK) . . . . . . . . . . . . . 102

5.5 .1 Problem: Schleifenbildung . . . . . . . . . . . . . . . . . . . . . . . . . . . . . . . . . . .

5.5 .2 Beispiel: MIK AT-Geräte-Dienste . . . . . . . . . . . . . . . . . . . . . . 104

5.6 Überwachung der Umgebung - Remote-Model-Inspektor (RMI) . . . . . . . . . . 104

5.7 Verwaltung der Dienste und Geräte - Dienst/Geräte-Inspektor (DGI) . . . . . . . 106

5.8 Migration von traditioneller Datenhaltung zur Repräsentation der Information in einer verteilten, modellgetriebenen Instanzumgebung . . . . . . . . . . . . 107

5.9 Prototypen der Komponenten . . . . . . . . . . . . . . . . . . . . . . . . 108

6 Anwendungen: Dynamik auf Basis der verteilten, modellgetriebenen Ausführungsumgebung $\quad 110$

6.1 Verteilung in der Automatisierungstechnik als Suche . . . . . . . . . . . . . . 110

6.2 Migration von Komponenten . . . . . . . . . . . . . . . . . . . . . . . . 112

6.3 Anwendungsfall 1: Abbildung einer Remote $1 / 0 \ldots \ldots$. . . . . . . . . . . . . 114

6.4 Anwendungsfall 2: Vorbereitung auf Ausfälle . . . . . . . . . . . . . . . . . . 115

6.5 Anwendungsfall 3: Adressierung durch PLT-Stelle . . . . . . . . . . . . . . . . . 116

6.6 Anwendungsfall 4: IEC61131-3-Programmierung im Modell . . . . . . . . . . . . 117

6.6.1 Probleme der konsequenten Umsetzung . . . . . . . . . . . . . . . . 118

7 Zusammenfassung $\quad 120$

7.1 Ausblick . . . . . . . . . . . . . . . . . . . . 121

$\begin{array}{lr}\text { Begriffsverzeichnis } & 122\end{array}$

$\begin{array}{lr}\text { Literaturverzeichnis } & 125\end{array}$

$\begin{array}{lr}\text { Normen und Richtlinien } & 128\end{array}$ 


\section{Kurzfassung}

Für die Weiterentwicklung der Automatisierungstechnik ist die erweiterte Zusammenarbeit der automatisierungstechnischen Geräte wichtig. Dieses gilt für alle Phasen einer Anlage: Von der Planung über die Produktion bis zur Wartung. Ebenso auch für die horizontale und vertikale Integration während der Produktion. Viele aktuelle Themen, wie „Industrie 4.0“ oder „Cyber Physical Systems“ gehen davon aus, dass Informationen aus dem Engineering zur Produktionszeit bereit stehen. Hierzu leistet diese Arbeit einen Beitrag.

Informationen werden heutzutage noch häufig entweder nicht elektronisch auswertbar gespeichert (beispielsweise als Grafiken) oder sind so abgelegt, dass nur einzelne Programme auf sie zugreifen können. Hierdurch sind die existierenden Informationen nicht so weit zugreifbar, wie sie es eigentlich sein könnten.

Modelle spielen hierbei eine entscheidende Rolle: Sie beschreiben Sachverhalte der Anlagen. Die meisten der heutigen Modelle werden bei ihrer Spezifikation in einem elektronisch abbildund auswertbaren Format definiert, sodass ein Computer die Informationen sowohl bereitstellen, wie auch auswerten und damit nutzen kann. Werden diese Modelle zur Produktionszeit bereitgestellt und genutzt, werden hierdurch dynamische Änderungen ermöglicht, die heutzutage nicht üblich sind. In der Automatisierungstechnik wird deswegen immer mehr angestrebt, Modelle zur Beschreibung der unterschiedlichsten Sachverhalte zu nutzen. Modelle beschreiben unter anderem Systemstrukturen einer Anlage. Dabei werden Themen-spezifische Modelle entwickelt, die unabhängig voneinander jeweils eigene Blickwinkel der Automatisierungstechnik auf eine Anlage beschreiben.

Im Gegensatz dazu wurden Versuche, bei denen eine Domäne als Ganzes (wie beispielsweise Automatisierungstechnik) abgebildet werden soll, nicht von Erfolg gekrönt.

Die Modelle konnten sich beispielsweise nicht etablieren, weil eine Verbreitung nicht erreicht wurde. Dieses mag insbesondere daran gelegen haben, dass die jeweiligen Detaillierung von umfassenden Modellen (sogenannten „Welt-Modellen“) für spezifische Anwendungsfälle nicht ausreichend waren. Da diese Modelle in dem Fall nicht eingesetzt werden konnten, wurde wiederum auf Eigenentwicklungen gesetzt, was den Bestrebungen des Welt-Modells widersprach. Die vorliegende Arbeit beschreibt Mittel, um die gleichen Ziele zu erreichen, aber auf ein zentralistisches Modell zu verzichten: Existierende, heterogene Modelle werden in allen Phasen und Ebenen einer verteilten Umgebung, wie in einem automatisierungstechnischen System, nutzbar gemacht.

Hierzu wird zum einen eine verteilte, dynamische modellgetriebene Instanzumgebung beschrieben. Sie kann als Erweiterung von existierenden Technologien begriffen werden, wird aber unabhängig von diesen dargestellt. Durch diese modellgetriebene Instanzumgebung wird erreicht, 
Teile eines Modells auf unterschiedlichen Geräten bereitzustellen. Diese sind in einer einheitlichen Weise abfragbar und erkundbar. Hierdurch können die im Modell abgebildeten Systemstrukturen an einem „sinnvollen“ Ort abgelegt werden, der nach Kriterien, wie der häufigsten Nutzung, der höchsten Ausfallsicherheit oder der schnellsten Verfügbarkeit beim Zugriff erfolgen kann. Gleichzeitig bietet eine solche Ausführungsumgebung der Modelle die Möglichkeit dynamisch auf Änderungen zu reagieren: kollaborativ erfolgen Änderungen von unterschiedlichen Anwendungen. Dabei muss jedoch insbesondere die Transaktionssicherheit sowie Nachvollziehbarkeit (Determinismus) der Änderungen gegeben sein.

Zum anderen beschreibt die Arbeit ein Konzept zur Interkonnektion von Modellen. Hierbei werden Teile von Modellen, die unabhängig voneinander entworfen wurden, in Relation zueinander gesetzt. Interkonnektionen stellen dabei eine Form von Relationen dar, die jedoch keinerlei Abhängigkeit an Ausgangs- sowie Zielpunkt voraussetzen. Dieses erlaubt die Modellierung von zusätzlichen Sachverhalten, sodass die Interkonnektion von Modellen wiederum ein Modell ist.

Durch die Kombination dieser beiden Aspekte ergibt sich eine verteilte, deterministische und dynamische Ausführungsumgebung für Systemstrukturen. Voraussetzung ist ein gemeinsames Meta-Modell sowie Verständnis der Problematik. Als Folge können Modelle unabhängig voneinander entworfen werden. Eine solche Ausführungsumgebung muss dabei Schnittstellen bereitstellen, um die Informationen abzufragen und entsprechende Änderungen vorzunehmen. Erst hierdurch können aufbauende Anwendungen einen realen Nutzen aus den Konzepten ziehen.

Insgesamt ergibt sich so eine Lösung, um Modelle zu den unterschiedlichen Phasen und Ebenen einer Anlage - insbesondere auch zur Produktionszeit - zu nutzen. Die Integration der verteilten, unterschiedlichen Modelle beschreibt die informationstechnische Basis, um dynamisch auf Änderungen in den Systemstrukturen zu reagieren. Hierunter werden beispielsweise Änderungen der Anlagenstruktur durch Umbauten ebenso verstanden, wie unterschiedliche Produktionsaufträge. 


\section{Abstract}

For the future development of automation technology the enhanced collaboration of automation devices is important. This is true for all phases of a plant, from planning to production to maintenance, as well as for the horizontal and vertical integration during the production. A lot of current topics like "Industry 4.0" or "Cyber Physical Systems" act on the assumption that engineering information is available during the production phase, which is not the case today. The dissertation addresses this topic.

Nowadays information is often stored not electronic analyzable (e.g. as graphic) or accessible only by single programs. So existing information is not accessible as far as it could be.

Models are acting as an important part: They describe characteristics of a plant. Most of today's models are defined in an electronically representable and evaluable format by their specification. So a computer could host and provide these models as well as evaluate and use them. If they are provided and used during the production phase, dynamical changes are made possible, which is not usually the case nowadays.

Therefore, in automation technology models are used for the description of different topics. Topic-specific models are developed, that are independent from each other and describe different aspects of the domain of automation technology.

In contrast attempts to describe the whole domain of automation technology in one model were not successful. Those models could not be widely established since their adoption could not be achieved. This could be due to the fact that models describing a whole domain ("World-Models") are not detailed enough to be used for specific cases. So specific models were required to be defined, which contradicts the purpose of whole domain model.

This work describes instruments without a centralized model: Existing, heterogeneous models can be used in all phases and levels of a distributed system like a plant in a homogenous way. Therefore a distributed, dynamic, model-driven execution environment is described. This could be seen as a further development of existing technologies, but is described independent of those. In this model-driven execution environment parts of a model could be provided by distributed devices. In a common way models are discover- and query-able. Therefore, information could be stored in a judicious place, that is defined by most frequent usage, highest reliability or fastest availability. At the same time such a model-driven execution environment provides for the possibility of dynamic changes: Changes are established in a collaborative way from different applications. For that purpose transaction security as well as comprehensibility (determinism) needs to be assured. 
Additionally, this work describes the concept of interconnections of models: Parts of models, which are designed independent of each other, are put in relation. Interconnections are a special type of relations not having dependencies at start- or endpoint. This enables modeling of additional aspects, so interconnections of models are models again.

This combination of instruments represents a distributed, deterministic and dynamic model execution environment of system structures. A requirement for this is a common used metamodel as well as a complete understanding of the topic. Models can be specified independent of each other. A model execution environment will provide interfaces for querying information and for making changes to all models. Applications will be based on this.

The overall result is a solution, which makes the use of models feasible during all phases of a plant - especially during production time. The integration of the distributed models provides an information-technology foundation for dynamic changes on system architecture. This covers changes of plants due to rebuilding as well as production orders. 
https://doi.org/10.51202/9783186245083-1

Generiert durch IP '172.22.53.54', am 26.04.2023, 04:11:05

Das Erstellen und Weitergeben von Kopien dieses PDFs ist nicht zulässig. 